\title{
Minor Planet Binaries
}

\author{
Jean-Luc Margot \\ California Institute of Technology, Pasadena, CA, USA
}

The first detailed characterization of a near-Earth binary (2000 DP107) provided significant insights into the formation mechanism, orbital evolution, and physical properties of the system. Near-Earth Asteroid (NEA) binaries are tidally evolved with synchronously rotating secondaries, providing constraints on asteroid mechanical properties (tidal $Q$ and rigidity). It is expected that radar observations will permit measurements of orbital precession, with corresponding implications for the distribution of mass within asteroids.

Several adaptive optics surveys of main-belt asteroid (MBA) binaries are underway. Apart from obtaining critical asteroid mass and density measurements, the goal is to measure the abundance of binary systems in that population and to constrain the collisional environment and the binary formation mechanism. MBA densities fall in the range 1.2-2.6, implying high porosity in main belt asteroids.

Binary Kuiper-Belt Objects (KBOs) are most likely primordial, and their abundance and properties constrain the environment in the early Kuiper belt. A carefully designed program combining ground-based observations and $25 \mathrm{HST}$ visits can provide accurate orbits for 6 binary KBO systems. Such observations are likely to give the first hints on the density and internal structure of distant ice-rock bodies.

I will describe binary systems in those populations, their physical properties, and formation mechanisms. 